\title{
Adaptive immunity against COVID-19
}

\author{
Umid Kumar Shrestha* \\ Nepal Mediciti Hospital, Bhaisepati, Lalitpur, Nepal
}

Keywords: COVID-19; SARS-CoV-2; adaptive immune response; neutralizing antibody; T-cell immunity

\section{Abstract}

The adaptive immune response has often been described as the key player in determining the severity of COVID-19 disease. The COVID-19 outcomes seem to be dependent upon the B-cell- and T-cell-mediated adaptive immunity to SARS-CoV-2. Coordinated CD4 ${ }^{+} \mathrm{T}$-cell, CD8 ${ }^{+}$T-cell, and antibody responses are protective, whereas, the uncoordinated immune responses are associated with the severe form of COVID-19. Multiple human vaccines in clinical trials to counteract SARS-CoV- 2 is based upon the neutralizing antibodies directed at the spike protein. The potential therapeutic and preventive monoclonal antibodies used in human clinical trials are also based on the viral neutralization. The vaccines can generate high levels of neutralizing antibodies comparable with or greater than those seen in sera samples from patients. The induction of sufficient CD4 ${ }^{+}$ helper T-cells and inclusion of vaccine boosts, may be needed to maintain levels of anti-SARS-CoV-2 neutralizing antibodies. The COVID-19 vaccines are in rapid development by utilizing the knowledge of adaptive immunity against COVID-19, and we are hopeful to get the effective and safe vaccines for the public use very soon.

in response to SARS-CoV-2, is depicted in the figure below.

\section{Editorial:}

Coronavirus disease 2019 (COVID-19) is caused by a novel Coronavirus now called severe acute respiratory syndrome coronavirus 2 (SARS-CoV-2) ${ }^{1}$ and the World Health Organization (WHO) has declared COVID-19 a global pandemic on March 11, $2020 .{ }^{2,3}$

The elderly patients, males more than females, smokers, and the patients with co-morbidities, such as hypertension, diabetes, cardiac disease, chronic kidney disease, obesity, immunocompromised state, liver disease, chronic underlying lung disease and cancer are more vulnerable for the severe COVID-19 disease. Several laboratory markers such as neutrophillymphocyte ratio, D-dimer, procalcitonin, ferritin, C-reactive protein (CRP), and pro-inflammatory interleukins can also help in predicting the severity of the disease. ${ }^{4}$

The immune response has often been described as the key player in determining the severity of COVID-19 disease.The COVID-19 outcomes seem to be dependent upon the B-celland T-cell-mediated adaptive immunity to SARS-CoV-2. ${ }^{5}$ Hence, the thorough knowledge of such adaptive immunity is important for developing effective strategies to control the pandemic. The adaptive immunity, which is developed

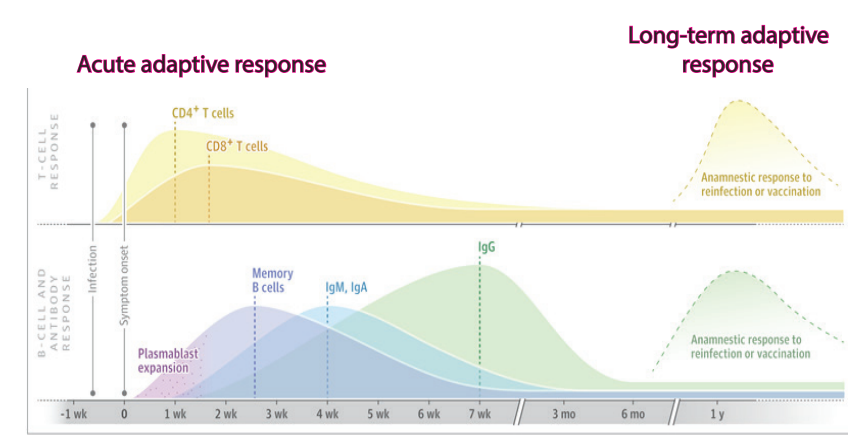

Figure: Adaptive immunity to COVID 2019: Generalized model of T-cell and B-cell (plasmablast, antibody) responses to SARS-CoV-2 infection projected over 1 year following infection; neutralizing antibodies, memory $\mathrm{B}$ cells, and $\mathrm{CD} 4^{+}$and $\mathrm{CD} 8^{+}$memory T-cells to SARS-CoV-2 are generated by infection, vaccination, or after re-exposure; the dotted lines represent peak B-cell, T-cell, and antibody responses following infection. ${ }^{5}$

*Corresponding Author:

Umid Kumar Shrestha, MD, PhD, FACG

Nepal Mediciti Hospital, Bhaisepati, Lalitpur, Nepal

E-mail: umidshrestha@gmail.com 
The disease severity (asymptomatic, mild, moderate, or severe) influences the magnitude of the antibody and T-cell responses among the different individuals. The neutralizing antibodies, especially those that recognize the viral receptor binding domain (RBD) and other epitopes on the spike protein that prevent subsequent angiotensin-converting enzyme II receptor binding, membrane fusion, and viral entry, are one of the main components of the adaptive immune response. The $\mathrm{CD} 4^{+} \mathrm{T}$-cell responses in convalescing patients correlates with the magnitude of anti-SARS-CoV-2 IgG and IgA titers to the spike protein; the viral neutralization correlates strongly with the magnitude of $\operatorname{lgG} 1$ and IgG3 RBD enzyme-linked immunosorbent assay (ELISA) titers. ${ }^{6,7}$

Multiple human vaccines in clinical trials to counteract SARS-CoV-2 is based upon the neutralizing antibodies directed at the spike protein. ${ }^{8}$ The potential therapeutic and preventive monoclonal antibodies used in human clinical trials are also based on the viral neutralization.

Upon the recovery of the disease, the patients with mild or asymptomatic disease have antibodies with low anti-RBD titers and low viral neutralizing activity. Antigen burden seems to be the major determinant of the magnitude of the immune response because the highest neutralizing antibody titers are found in the severe disease. ${ }^{7}$

One recent study examined the all three branches of adaptive immunity at the level of SARS-CoV-2-specific $C D 4^{+}$and $C D 8^{+}$T-cell and neutralizing antibody responses in acute and convalescent subjects. ${ }^{9}$ The study showed that the coordinated SARS-CoV2-specific adaptive immune responses were associated with milder disease, suggesting roles for both $\mathrm{CD}^{+}$and $\mathrm{CD} 8^{+} \mathrm{T}$-cells in protective immunity in COVID-19. The study also revealed that the coordination of SARS-CoV-2 antigen-specific responses was disrupted in individuals $\geq 65$ years old; the scarcity of naive T-cells was also associated with aging and poor disease outcomes. Therefore, it was concluded that the coordinated $\mathrm{CD}^{+} \mathrm{T}$-cell, $\mathrm{CD} 8^{+} \mathrm{T}$-cell, and antibody responses are protective, whereas, the uncoordinated immune responses are associated with the severe form of COVID-19. ${ }^{9}$

SARS-CoV-2-specific $\mathrm{CD}^{+}$and $\mathrm{CD}^{+}$memory T-cells are also generated in asymptomatic to severe disease. ${ }^{10-12}$ Individuals with mild or asymptomatic disease are reported to exhibit robust memory T-cell responses months after COVID-19 infection.

One study showed the presence of preexisting T-cell immunity to SARS-CoV-2 in blood donors either prior to the COVID-19 pandemic or more recently among those without infection. ${ }^{10-12}$ These memory T-cells (memory CD4 ${ }^{+}$T-cells found in higher frequencies than are $\mathrm{CD} 8^{+} \mathrm{T}$-cells) likely represent responses induced by previous infection with other human endemic betacoronaviruses known to cause the common cold. Such T-cells can recognize known or predicted epitopes within the nucleocapsid (N protein) and spike structural proteins as well as the nonstructural proteins (NSPs), NSP7 and NSP13. ${ }^{10,11}$ It is possible that the cross-reactive T-cells from previous coronavirus infections may be boosted by the exposure to SARS-CoV-2. These preexisting T-cells could influence the immune response to SARSCoV-2, affecting the ultimate COVID-19 outcome.This could help explain for the wide range of symptoms experienced by people with COVID-19. This indicates that the T-cells are another level of population-level immunity against COVID-19.

There was one recent study, which looked for the auto-antibodies against type I Interferon (IFN) to find out the reasons for the clinical variability in the course of SARS-CoV-2 infection..$^{13}$ The study showed that auto-antibodies against type I IFN were responsible for the life-threatening condition in some of the COVID-19 patients (they were found in $2.6 \%$ of women and $12.5 \%$ of men); the auto-antibodies seem to neutralize the ability of the corresponding type I IFNs to block SARS-CoV-2 infection. ${ }^{13}$

Another study has shown that the inborn errors of TLR3- and IRF7dependent type I IFN immunity [caused by the 'Loss-of-Function' (LOF) mutations of the genes at 13 loci] were responsible for the life-threatening condition in 3.5\% of COVID-19 patients, aged 17 to 77 years. ${ }^{14}$

The whole world is desperately waiting for the successful and safe COVID-19 vaccines, to be made available for the public. The vaccines can generate high levels of neutralizing antibodies comparable with or greater than those seen in sera samples from patients. The induction of sufficient $\mathrm{CD} 4^{+}$helper T-cells and inclusion of vaccine boosts, may be needed to maintain levels of anti-SARS-CoV-2 neutralizing antibodies. ${ }^{11}$ The COVID 19 vaccines are in rapid development by utilizing the knowledge of adaptive immunity against COVID 19, and we are hopeful to get the effective and safe vaccines for the public use very soon.

November 8, 2020.

\section{References}

1. CDC. 2019 Novel Coronavirus, Wuhan, China. Centers for Disease Control and Prevention. Available at https://www.cdc. gov/coronavirus/2019-ncov/about/index.html. 2020 Jan 26.

2. Gallegos A. WHO Declares Public Health Emergency for Novel Coronavirus. Medscape Medical News. Available at https:// www.medscape.com/viewarticle/924596. 2020 Jan 30.

3. Ramzy A, McNeil DG. W.H.O. Declares Global Emergency as Wuhan Coronavirus Spreads. The New York Times. Available at https://nyti.ms/2RER70M. 2020 Jan 30.

4. Udwadia ZU, Tripathi AR, Nanda VJ, et al. Prognostic factors for adverse outcomes in COVID-19 infection. J Assoc Physicians India 2020;68(7):56-60.

5. Stephens DS, McElrath MJ. COVID-19 and the Path to immunity. JAMA. 2020;324(13):1279-1281. doi:10.1001/ jama.2020.16656.

6. Suthar MS, Zimmerman MG, Kauffman RC, et al. Rapid generation of neutralizing antibody responses in COVID-19 patients. Cell Rep Med. 2020;1(3):100040. Published online June 23, 2020. doi:10.1016/j.xcrm.2020.100040.

7. Robbiani DF, Gaebler C, Muecksch F, et al. Convergent antibody responses to SARS-CoV-2 in convalescent individuals. Nature. Published online June 18, 2020. doi:10.1038/s41586-0202456-9.

8. O'Callaghan KP, Blatz AM, Offit PA. Developing a SARSCoV-2 vaccine at warp speed. JAMA. 2020; 324(5):437-438. doi:10.1001/jama.2020.12190.

9. Moderbacher CR, Ramirez SI, Dan JM, et al. Antigen-specific adaptive immunity to SARS-CoV-2 in acute COVID-19 and associations with age and disease severity. Cell 2020; (S00928674(20)31235-6). doi:10.1016/j.cell.2020.09.038.

10. Grifoni A, WeiskopfD, Ramirez SI, et al.Targets of T cell responses 
to SARS-CoV-2 coronavirus in Humans with COVID-19 disease and unexposed individuals. Cell. 2020;181(7):1489-1501.e15. doi:10.1016/j.cell.2020.05.015.

11. Le Bert N, Tan AT, Kunasegaran K, et al. SARS-CoV-2-specific T cell immunity in cases of COVID-19 and SARS, and uninfected controls. Nature 2020; 584, 457-462. doi:10.1038/s41586-020$2550-z$

12. Braun J, Loyal L, Frentsch $M$ et al. SARS-CoV-2-reactive T cells in healthy donors and patients with COVID-19. Nature 2020. Published online July 29, 2020. doi: 10.1038/s41586-0202598-9.

13. Bastard P, Rosen LB, Zhang Q. Auto-antibodies against type I IFNs in patients with life-threatening COVID-19. Science 2020. Published online on September 24, 2020; eabd4585. doi: 10.1126/science.abd4585.
14. Zhang Q, Bastard P, Liu Z, et al. Inborn errors of type I IFN immunity in patients with life-threatening COVID-19. Science 2020. Published online on September 24, 2020;eabd4570. doi: 10.1126/science.abd4570. 